\title{
Cytological features of programmed cell death in Nicotiana tabacum cells in relation to the expression and localization of death regulators.
}

Louise Brisson, Nathalie Bolduc, Mario Ouellet, Frédéric Pitre and Israel Fortin.

Department of Biochemistry and Microbiology, Life and Health Sciences Research Building, Laval University, Québec, Canada, G1K 7P4.

Plant cells could benefit from programmed cell death (PCD) in a number of developmental and physiological processes [1]. For example, during the establishment of the hypersensitive response (HR) PCD could play an important protecting role by limiting pathogen ingress. This death program is possibly regulated by a number of genes. To date, some regulators of plant PCD have been identified, but very few have been found to be homologous to animal PCD regulators [2]. Among these is the plant Bax Inhibitor-1 (BI-1) protein, which possesses, like its human counterpart, the ability to suppress the lethality induced by the proapoptotic regulator Bax [3-4]. Of interest, expression of BI-1 is rapidly upregulated in plant during wounding or pathogen challenge [4]. To increase our understanding of plant PCD and its mechanism of regulation, we have exploited genetic engineering to study the cytological features of Bax expression in mammalian and tobacco cells as well as to study the intracellular distribution of BI-1 protein in plant cells.

Cytological studies were conducted using transformation of human 293 cells, tobacco BY-2 cells or tobacco Xanthi leaves. Localization studies of Bax and BI-1 were investigated with the fusion proteins BaxGFP and BnBI-1GFP. Cells transformed with untargeted GFP, or GFP targeted to the ER were used as controls. Compared to non transformed cells, murine Bax expression was associated with an increased accumulation of mammalian and tobacco dead cells. This Bax-induced death was reduced by co-transforming mammalian cells with Bcl-2 or with BI-1 indicating that both plant BI-1 homologues and Bcl-2 can suppress Baxinduced apoptosis at a similar level (Figure 1). Production of Bax and Bcl-2 in transfected cells was attested by immunoblot analysis (Figure 1).

BY-2 cells expressing GFP alone or in fusion to Bax showed diffuse fluorescence distributed throughout the cytoplasm and in the nucleoplasm (Figure 2A). Bax-induced-cell death was frequently accompanied by typical "apoptosis"-related changes in nuclear morphology. In BY-2 cells expressing BnBI-1 GFP, the fluorescence was mainly observed in the vicinity of the nuclear envelope and within endoplasmic reticulum (data not shown). Of interest, fluorescence of BY-2 cells expressing BnBI-1-GFP or ER-GFP was localized to a number of spherical vesicles after an exposure to $125 \mathrm{mM} \mathrm{SA}$, which was markedly different to the distribution observed in untreated cells (data not shown). Taken together, our 
observations suggest that regulators of cell death could operate in diverse organisms indicating that this process is functionally conserved throughout the evolution.

[1] A. Danon et al., Plant Physiol. Biochem 38 (2000) 647.

[2] R.I. Pennell and C. Lamb, Plant Cell 9 (1997) 1157.

[3] M. Kawai et al., FEBS Lett. 464 (1999) 143.

[4] P. Sanchez et al., Plant J. 21 (2000) 393.

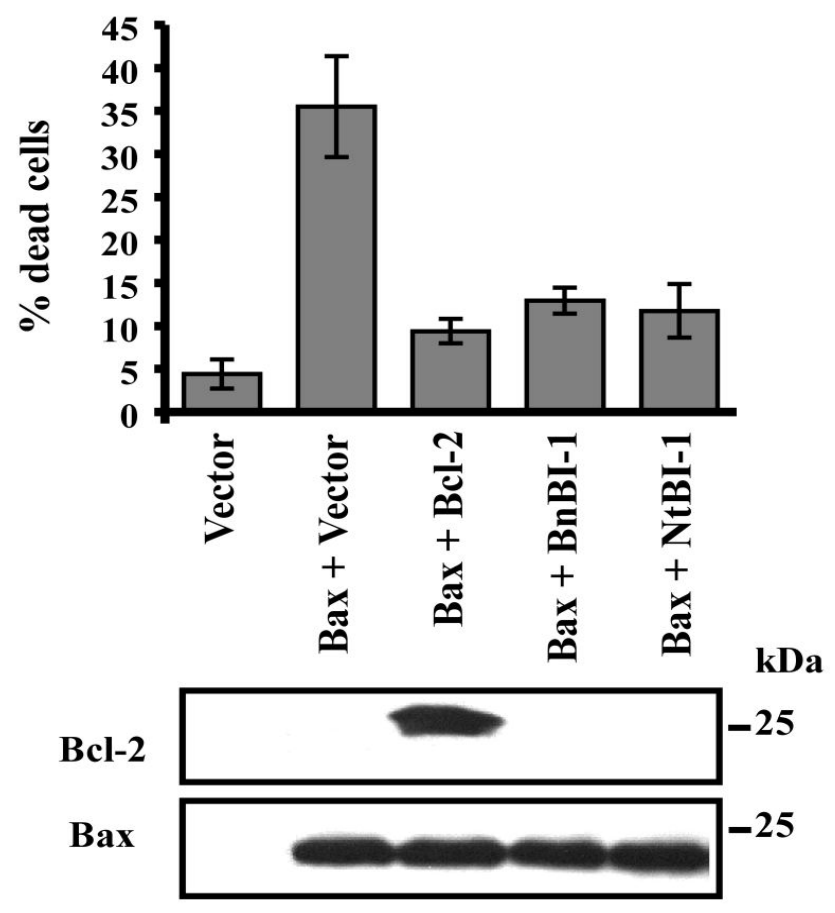

Figure1. Death assay in human 293 cells. Cells were either transfected with control vector, or cotransfected with Bax plasmid together with control vector or plasmids encoding Bcl-2, BnBI-1 or NtBI-1. Dead cells were determined using trypan blue dye exclusion assay (top). Protein extracts from a fraction of transfected cells were subjected to immunodetection using antibodies specific for Bcl-2 and murine Bax proteins (bottom).
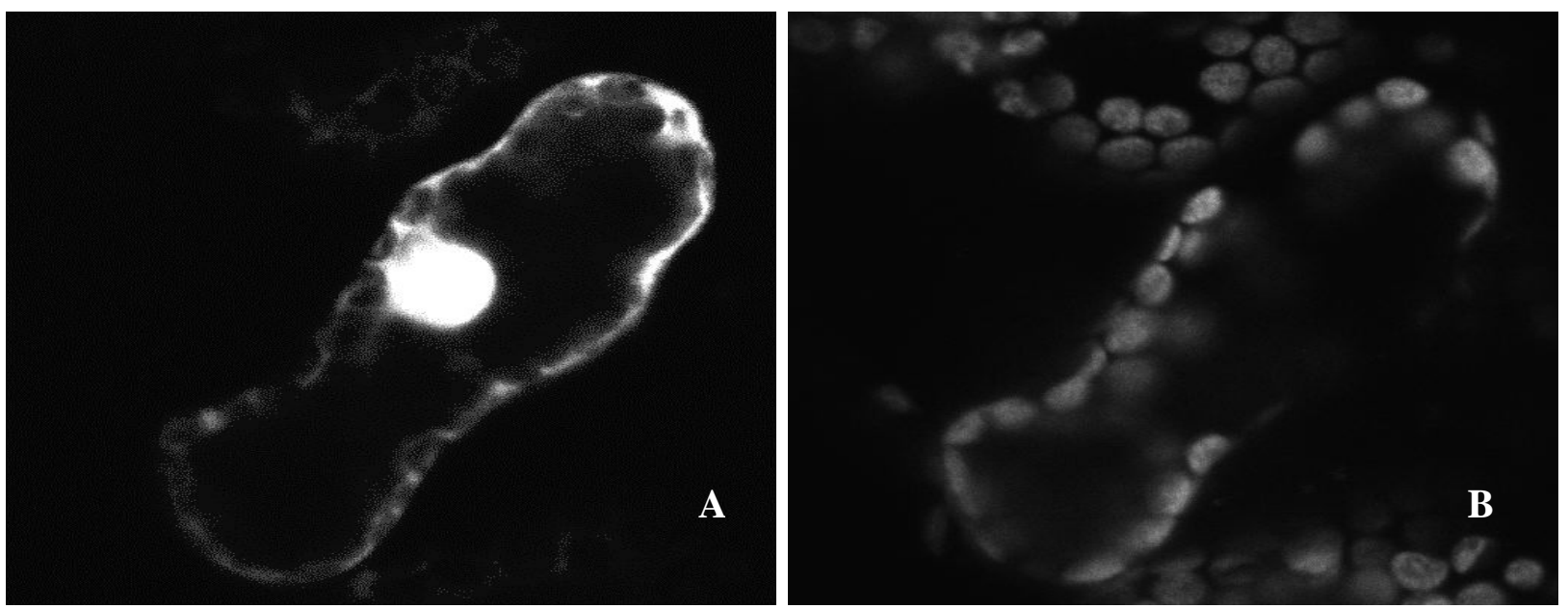

Figure 2. Transient Bax expression in palisade mesophyll tobacco cells. Transformed plant cells were viewed under a confocal microscope at an excitation wavelength of $488 \mathrm{~nm}$ (A) and $543 \mathrm{~nm}$ (B) to view the fluorescence of GFP and chlorophyll, respectively. 